TRANSACTIONS OF THE

AMERICAN MATHEMATICAL SOCIETY

Volume 365, Number 12, December 2013, Pages 6499-6515

S 0002-9947(2013)05925-9

Article electronically published on April 25, 2013

\title{
ON THE QUANTIZATION OF SPHERICAL NILPOTENT ORBITS
}

\author{
LIANG YANG
}

\begin{abstract}
Let $G$ be the real symplectic group $\operatorname{Sp}(2 n, \mathbb{R})$. This paper determines the global sections of certain line bundles over the spherical nilpotent

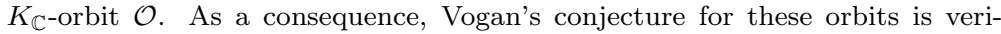
fied. The conjecture holds that there exists a unique unitary $(\mathfrak{g}, K)$-module structure on the space of the algebraic global sections of the line bundle associated to the admissible datum, provided that the boundary $\partial \overline{\mathcal{O}}$ has complex codimension at least 2 in $\overline{\mathcal{O}}$. Similar results are obtained for the metaplectic twofold cover $M p(2 n, \mathbb{R})$ of $S p(2 n, \mathbb{R})$.
\end{abstract}

\section{INTRODUCTION}

Orbit method plays an important role in representation theory of Lie groups. Kirillov used it to classify the unitary dual of simply connected nilpotent Lie groups. After that Kostant and Auslander later classified the unitary dual of simply connected solvable Lie groups of type I. Both classifications indicated that there should exist a correspondence between the coadjoint orbits and the unitary dual. However Duflo defined a subset of coadjoint orbits called admissible coadjoint orbits, which he found were more closely related to the unitary dual of reductive Lie groups. Later, Vogan intended to reduce these orbits to nilpotent coadjoint orbits, the quantization of which then became the key issue (see 22]). This paper tries to quantize the spherical nilpotent coadjoint orbits, in the hope that it could shed new light on the orbit method of real reductive Lie groups.

Let $G$ be a connected real reductive Lie group in Harish-Chandra's class with Lie algebra $\mathfrak{g}_{0}$. Then the nilpotent coadjoint $G$-orbits in $\mathfrak{g}_{0}^{*}$ can be identified with the nilpotent adjoint $G$-orbits in $\mathfrak{g}_{0}$ by a nondegenerate $\mathfrak{g}_{0}$-invariant bilinear form. Let $K$ be the maximal compact subgroup of $G$, and $K_{\mathbb{C}}$ be the complexification of $K$. According to Kostant-Sekiguchi correspondence, the nilpotent $G$-orbits in $\mathfrak{g}_{0}$

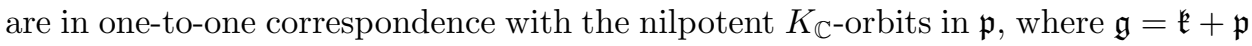
is the complexified Cartan decomposition of $\mathfrak{g}_{0}$.

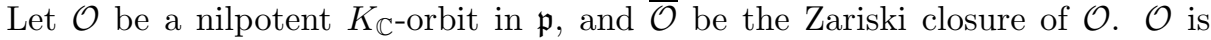
called a Vogan orbit if the boundary $\partial \overline{\mathcal{O}}$ has complex codimension at least 2 in $\overline{\mathcal{O}}$. This paper adopts Vogan's definition of admissible orbits, while Duflo's definition as mentioned above is equivalent to Vogan's definition of nilpotent $K_{\mathbb{C} \text {-orbits }}$ (see 20]). Let $(x, \chi)$ be an admissible datum of $\mathcal{O}$ (see Section 2). Then we can define

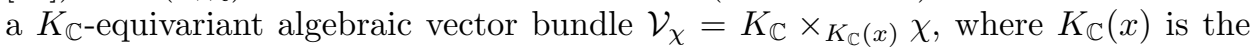

Received by the editors November 9, 2011 and, in revised form, May 20, 2012.

2010 Mathematics Subject Classification. Primary 20G15, 22E46.

Key words and phrases. Admissible data, spherical nilpotent orbits, Vogan's conjecture.

Part of this work was included in the author's Ph.D. thesis.

(C) 2013 American Mathematical Society Reverts to public domain 28 years from publication 
stabilizer of $x$ in $K_{\mathbb{C}}$. The following conjecture describes the unitary representation "attached" to $(x, \chi)$.

Vogan's conjecture [20, see also [1, Conjecture 2.9]. Let $G$ be a real reductive Lie group, and $(x, \chi)$ be a nilpotent admissible datum. Assume, in addition, that the nilpotent orbit $\mathcal{O}=K_{\mathbb{C}} \cdot x$ is a Vogan orbit. Then there exists a compatible $\mathfrak{g}$ action on the spaces of algebraic sections of $\mathcal{V}_{\chi}$ such that $\Gamma\left(\mathcal{O}, \mathcal{V}_{\chi}\right)$ carries a unitary $(\mathfrak{g}, K)$-module structure.

In [6], Huang and Li study the associated varieties of the representations obtained by theta lifting. Combining it with the classification of low rank representations, they prove that the representations, whose associated varieties are the closure of spherical orbits, should be obtained by theta lifting. In [15, Nishiyama, Ochiai, Taniguchi and Yamashita find the irreducible unitary representations attached to the holomorphic orbits by theta lifting. All these findings indicate that the unitary $(\mathfrak{g}, K)$-module structure conjectured by Vogan should be obtained by theta lifting.

Given a nilpotent $K_{\mathbb{C}}$-orbit $\mathcal{O}$ in $\mathfrak{p}$, there is a Kostant-Sekiguchi triple (Definition 2.2) $\{x, h, y\}$ attached to $\mathcal{O}$. The adjoint action ad $h$ on $\mathfrak{g}$ gives a grading on $\mathfrak{g}$,

$$
\mathfrak{g}(m)=\{z \in \mathfrak{g} \mid[h, z]=m z\}, \mathfrak{k}(m)=\mathfrak{g}(m) \cap \mathfrak{k}, \mathfrak{p}(m)=\mathfrak{g}(m) \cap \mathfrak{p} .
$$

We define a parabolic subalgebra $\mathfrak{q}=\mathfrak{l}+\mathfrak{u} \subset \mathfrak{k}$ by

$$
\mathfrak{q}=\sum_{m \geq 0} \mathfrak{k}(m), \mathfrak{u}=\sum_{m>0} \mathfrak{k}(m), \mathfrak{l}=\mathfrak{k}(0) .
$$

Let $Q=L_{\mathbb{C}} U$ be the corresponding parabolic subgroup of $K_{\mathbb{C}}$. Moreover, the $\mathfrak{g}$-height of $\mathcal{O}$ is defined as the largest $m$ such that $\mathfrak{g}(m)$ is nonzero.

Let $G$ be the real symplectic group $S p(2 n, \mathbb{R})($ or $M p(2 n, \mathbb{R}))$ and $\mathcal{O}$ be a spher-

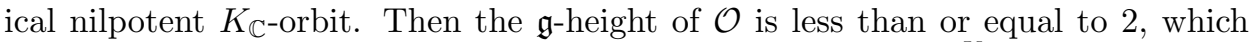
makes it possible to determine the space of global sections $\operatorname{Ind}_{K_{\mathbb{C}}(x)}^{K_{\mathbb{C}}} \chi$ of the equivariant line bundle associated to the admissible datum. The approach adopted here is induction by stages. To be more precise, since $K_{\mathbb{C}}(x) \subset Q \subset K_{\mathbb{C}}$, it can be deduced that

$$
\operatorname{Ind}_{K_{\mathbb{C}}(x)}^{K_{\mathbb{C}}} \chi \simeq \operatorname{Ind}_{Q}^{K_{\mathbb{C}}} \operatorname{Ind}_{K_{\mathbb{C}}(x)}^{Q} \chi
$$

Frobenius reciprocity and Kostant's Borel-Weil theorem are used to calculate $\operatorname{Ind}_{Q}^{K_{\mathbb{C}}}$ and $\operatorname{Ind}_{K_{\mathbb{C}}(x)}^{Q}$. In particular, this method could be used to compute the ring of regular functions on $\mathcal{O}$. Then the focus is on the unitary $(\mathfrak{g}, K)$-module structure conjectured by Vogan. Let $(O(p, q), S p(2 n, \mathbb{R}))$ be a dual pair in a stable range with $O(p, q)$ the smaller member. After determining the $K$-types of the theta lifting $\theta(\xi)$ of the unitary character $\xi$ of $\widetilde{O(p, q)}$, a comparison of the $K$-module structure of $\operatorname{Ind}_{K_{\mathbb{C}}(x)}^{K_{\mathbb{C}}} \chi$ and $\theta(\xi)^{*}$ leads to the following theorem.

Theorem (Theorem 4.8). Let $\mathcal{O}$ be an admissible Vogan orbit of $\operatorname{Sp}(2 n, \mathbb{R})$ (or $M p(2 n, \mathbb{R})$ ) corresponding to $(+2)^{p}(-2)^{q}(+1)^{2(n-p-q)}$, the signed Young diagram. Then $n>p+q$. Let $(x, \chi)$ be an admissible datum of $\mathcal{O}$. Then there exists a unitary representation $\theta(\xi)$, which is the theta lifting of a unitary character $\xi$ of $\widetilde{O(p, q)}$, such that

$$
\left.\operatorname{Ind}_{K_{\mathbb{C}}(x)}^{K_{\mathbb{C}}} \chi \simeq(\theta(\xi))^{*}\right|_{K}
$$

and the associated variety $\mathcal{A} \mathcal{V}\left((\theta(\xi))^{*}\right)=\overline{\mathcal{O}}$. If $(p, q) \neq(1,1)$, this representation is unique up to isomorphism. 
If $\operatorname{dim}_{\mathbb{C}} \mathcal{O}-\operatorname{dim}_{\mathbb{C}} \partial \mathcal{O}=1, \mathcal{O}$ is not a Vogan orbit. It is believed that the representation obtained by theta lifting should be the one attached to $\mathcal{O}$, since $\left.\pi(x, \chi)\right|_{K} \hookrightarrow \operatorname{Ind}_{K_{\mathbb{C}}(x)}^{K_{\mathbb{C}}} \chi$ (see [16]). Moreover, these results can be generalized to other real classical groups of type I.

In 2, Binegar tries to realize these representations by the degenerate principal series. In 12 and [13, Lee and Zhu prove that some of these representations could be embedded into the degenerate principal series.

This paper consists of four parts. Section 1 is the introduction. Section 2 reviews

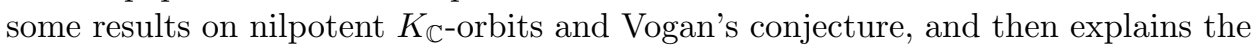
author's method of calculating the global sections of $\mathcal{V}_{\chi}=K_{\mathbb{C}} \times_{K_{\mathbb{C}}(x)} \chi$. Section 3 contains general results of dual pairs. The main results are shown in Section 4.

\section{Global SECTiOns of Line BUndLES OVER NiLPotent orbits}

This section begins with some results on nilpotent orbits for real reductive Lie groups in Harish-Chandra's class. Then Vogan's definition on admissible nilpotent $K_{\mathbb{C}^{-} \text {-orbits }}$ and Vogan's conjecture are introduced. Then the explanation of the author's method of computing the admissible datum and the global sections of a line bundle associated to it follows.

2.1. Nilpotent orbits and Vogan's conjecture. Let $G$ be a real reductive Lie group in Harish-Chandra's class. Let $\mathfrak{g}_{0}$ stand for the Lie algebra of $G$ and $\mathfrak{g}$ for its complexification. Analogous notation will be used for other groups. Fix a Cartan involution $\theta$ of $G$, then the fixed points $K=G^{\theta}$ is a maximal compact subgroup of $G$. We write $\mathfrak{g}_{0}=\mathfrak{k}_{0}+\mathfrak{p}_{0}$ for its Cartan decomposition and $\mathfrak{g}=\mathfrak{k}+\mathfrak{p}$ for the complexified Cartan decomposition. Now extend $\theta$ to $\mathfrak{g}$ as a complex linear involution, and define $\sigma$ to be the conjugation of $\mathfrak{g}$ with respect to $\mathfrak{g}_{0} . K_{\mathbb{C}}$ is written for the complexification of $K$.

Consider the adjoint action of $G$ on $\mathfrak{g}_{0}$. An element $x^{\prime} \in \mathfrak{g}_{0}$ is called nilpotent if $r x^{\prime} \in G \cdot x^{\prime}$ for all $r>0$. Nilpotent elements in $\mathfrak{p}$ can be defined in similar ways. Let $\mathcal{N}_{\mathfrak{g}_{0}}, \mathcal{N}_{\mathfrak{p}}$ denote the set of nilpotent elements of $\mathfrak{g}_{0}$ and $\mathfrak{p}$ respectively. $\mathcal{N}_{\mathfrak{g}_{0}} / G, \mathcal{N}_{\mathfrak{p}} / K_{\mathbb{C}}$ will denote the nilpotent orbits in $\mathfrak{g}_{0}$ and $\mathfrak{p}$ under the action of $G$ and $K_{\mathbb{C}}$ respectively.

Let $x^{\prime} \in \mathfrak{g}_{0}$ be a nilpotent element; then there exists a standard triple $\left\{x^{\prime}, h^{\prime}, y^{\prime}\right\}$ $\subset \mathfrak{g}_{0}$, i.e., $\left[h^{\prime}, x^{\prime}\right]=2 x^{\prime},\left[h^{\prime}, y^{\prime}\right]=-2 y^{\prime},\left[x^{\prime}, y^{\prime}\right]=h^{\prime}$. We call $x^{\prime}$ the nilpositive element of this $\mathfrak{s l}_{2}$-triple.

Fixing a Cartan involution $\theta$, one can show any standard triple $\left\{x^{\prime}, h^{\prime}, y^{\prime}\right\} \subset \mathfrak{g}_{0}$ is conjugate to another triple $\{x, h, y\}$ such that $\theta(h)=-h, \theta(x)=-y$. Triples with this property are called Cayley triples.

Given a Cayley triple $\left\{x^{\prime}, h^{\prime}, y^{\prime}\right\}$, let

$$
x=\frac{1}{2}\left(x^{\prime}+y^{\prime}+i h^{\prime}\right), h=i\left(x^{\prime}-y^{\prime}\right), y=\frac{1}{2}\left(x^{\prime}+y^{\prime}-i h^{\prime}\right) .
$$

Then $\{x, h, y\}$ is a standard triple in $\mathfrak{g}$, which is called the Cayley transformation of $\left\{x^{\prime}, h^{\prime}, y^{\prime}\right\}$. It follows immediately that $h \in \mathfrak{k}$ and $x, y \in \mathfrak{p}$. Any standard triple in $\mathfrak{g}$ with this property is called normal.

Theorem 2.1 ([3, Theorem 9.5.1). There is a natural one-to-one correspondence

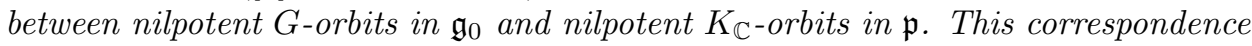
sends the zero orbit to the zero orbit and the orbit through the nilpositive element of a Cayley triple to the one through the nilpositive element of its Cayley transform. 
Definition 2.2 (Kostant-Sekiguchi triples). A standard triple $\{x, h, y\} \subset \mathfrak{g}$ is said to be a Kostant-Sekiguchi triple if $h \in \mathfrak{k}, x, y \in \mathfrak{p}$ and $\sigma(x)=y$.

We write $\Lambda=\left(+m_{1}\right)^{r_{1}^{+}}\left(-m_{1}\right)^{r_{1}^{-}} \ldots\left(+m_{d}\right)^{r_{d}^{+}}\left(-m_{d}\right)^{r_{d}^{-}}$for the signed Young diagram. The factor $\left(+m_{i}\right)^{r_{i}^{+}}$denotes for $r_{i}^{+}$rows with length $m_{i}$ in $\Lambda$, beginning with

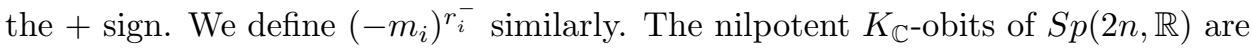
classified in the following theorem.

Theorem 2.3 ([3], Theorem 9.3.5). The nilpotent orbits in $\mathfrak{s p}(2 n, \mathbb{R})$ are parameterized by signed Young diagrams of size $2 n$ and any signature in which odd rows begin with + and occur with even multiplicity.

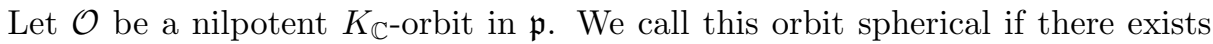
an open $B$-orbit in $\mathcal{O}$, where $B$ is a Borel subgroup of $K_{\mathbb{C}}$. One can also prove that

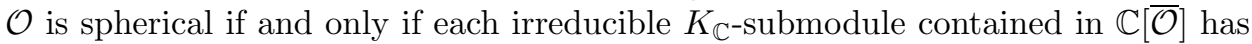
multiplicity one. The spherical nilpotent $K_{\mathbb{C}^{-o r b i t s}}$ in $\mathfrak{p}$ are classified by D. King in 10 .

Theorem 2.4 ([10, Theorem 6.1$)$. The spherical nilpotent $K_{\mathbb{C}}$-orbits of $S p(2 n, \mathbb{R})$ are precisely those corresponding to signed Young diagrams $\Lambda$ such that each row has length less than or equal to 2.

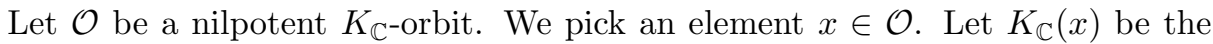
stabilizer of $x$. Then $\mathcal{O}=K_{\mathbb{C}} / K_{\mathbb{C}}(x)$. Define a character

$$
\gamma(x): K_{\mathbb{C}}(x) \rightarrow \mathbb{C}^{\times}, \gamma(x)(k)=\operatorname{det}\left(\left.\operatorname{Ad}(k)\right|_{(\mathfrak{k} / \mathfrak{k}(x))^{*}}\right) .
$$

A representation $\chi$ of $K_{\mathbb{C}}(x)$ is called admissible if the differential of $\chi$ equals $\frac{1}{2} \mathrm{~d} \gamma(x)$, i.e.,

$$
\chi(\exp A)=\gamma(x)\left(\exp \left(\frac{A}{2}\right)\right) \cdot I, A \in \mathfrak{k}(x) .
$$

An admissible datum is a pair $(x, \chi)$ where $\chi$ is an irreducible admissible represen-

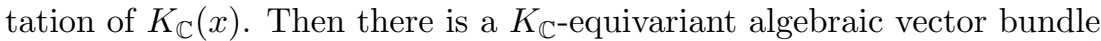

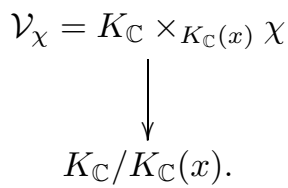

The nilpotent orbit $\mathcal{O}=K_{\mathbb{C}} / K_{\mathbb{C}}(x)$ is called admissible if the admissible orbit data $(x, \chi)$ exists. In fact, this is not the original definition of admissibility by Duflo, but J. Schwartz proved that this is equivalent to Duflo's definition (see [20]). Given an admissible datum, Vogan has a conjecture to predict the $K$-types of the quantization.

Conjecture 2.5 (Vogan's conjecture [20, see also [1], Conjecture 2.9). Let G be a real reductive Lie group, and $(x, \chi)$ be a nilpotent admissible datum. Assume, in addition, that the boundary of the orbit closure $\overline{K_{\mathbb{C}} \cdot x}$ has complex codimension at least 2. Let $\pi(x, \chi)$ be the unitary representation of $G$ conjecturally attached to $x$ and $\chi$. Then the space of $K$-finite vectors of $\pi(x, \chi)$ is isomorphic to the spaces of algebraic sections of $\mathcal{V}_{\chi}$.

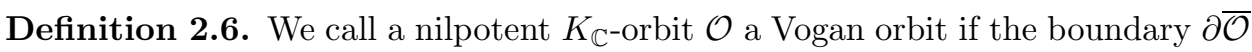
has complex codimension at least 2 in $\overline{\mathcal{O}}$. 


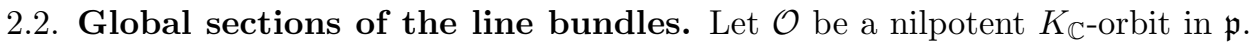
Then there exists a Kostant-Sekiguchi triple $\{x, h, y\}$ such that $\mathcal{O}=K_{\mathbb{C}} \cdot x$. The adjoint action ad $h$ on $\mathfrak{g}$ gives a grading on $\mathfrak{g}$,

$$
\mathfrak{g}(m)=\{z \in \mathfrak{g} \mid[h, z]=m z\}, \mathfrak{k}(m)=\mathfrak{g}(m) \cap \mathfrak{k}, \mathfrak{p}(m)=\mathfrak{g}(m) \cap \mathfrak{p} .
$$

We define the $\mathfrak{g}$-height (resp. $\mathfrak{k}$-height, $\mathfrak{p}$-height) of $\mathcal{O}$ to be the largest $m$ such that $\mathfrak{g}(m)$ (resp. $\mathfrak{k}(m), \mathfrak{p}(m))$ is nonzero. With this grading, we define a parabolic subalgebra $\mathfrak{q}=\mathfrak{l}+\mathfrak{u} \subset \mathfrak{k}$ by

$$
\mathfrak{q}=\sum_{m \geq 0} \mathfrak{k}(m), \mathfrak{u}=\sum_{m>0} \mathfrak{k}(m), \mathfrak{l}=\mathfrak{k}(0) .
$$

Let $Q=L_{\mathbb{C}} U$ be the corresponding parabolic subgroup of $K_{\mathbb{C}}$, here

$$
L_{\mathbb{C}}=\left\{k \in K_{\mathbb{C}} \mid \operatorname{Ad}(k) h=h\right\},
$$

and let $U$ be the connected subgroup in $K_{\mathbb{C}}$ with Lie algebra $\mathfrak{u}$. One proves (see [1]) that if $h$ satisfies the condition $\sigma(h)=-h, L_{\mathbb{C}}$ is the complexification of the compact group

$$
L=\{k \in K \mid \operatorname{Ad}(k) h=h\} .
$$

We write $L(x)$ for the stabilizer of $x$ in $L$, and $L_{\mathbb{C}}(x)$ for the stabilizer of $x$ in $L_{\mathbb{C}}$. If $\{x, h, y\}$ is a Kostant-Sekiguchi triple, $L_{\mathbb{C}}(x)$ is the complexification of $L(x)$ by Proposition 4.5 in 23 .

Proposition 2.7 ([1], Proposition 3.2). With the above setting, write $K_{\mathbb{C}}(x)$ for the stabilizer of $x$ in the adjoint action. There is a Levi decomposition $K_{\mathbb{C}}(x)=$ $L_{\mathbb{C}}(x) U(x)$. In particular, this isotropy group is contained in $Q$.

If the nilpotent $K_{\mathbb{C}^{-} \text {orbit has }}$-height less than or equal to 2 , the nilpotent radical $\mathfrak{u}$ is contained in $\mathfrak{k}(x)$, and $U=U(x)$. Then we have the following proposition.

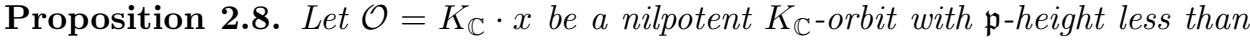
or equal to 2 . Let $\chi$ be a character of $K_{\mathbb{C}}(x)=L_{\mathbb{C}}(x) U$ with trivial $U$-action. Then $\operatorname{Ind}_{K_{\mathbb{C}}(x)}^{Q} \chi$ is completely reducible as a $Q$-module, and we can identify it with the trivial extension of an $L_{\mathbb{C}}$-module.

Proof. Given any $f \in \operatorname{Ind}_{K_{\mathbb{C}}(x)}^{Q} \chi$, then $f$ is an algebraic map from $Q$ to $\mathbb{C}$ such that $f(z g)=\chi^{-1}(g) f(z)$ for all $z \in Q$ and $g \in K_{\mathbb{C}}(x)$. For any $u \in U, f(u z)=$ $f\left(z\left(z^{-1} u z\right)\right)$. Since $U$ is a normal subgroup of $Q, z^{-1} u z \in U$,

$$
f\left(z\left(z^{-1} u z\right)\right)=\left(\chi\left(z^{-1} u z\right)\right)^{-1} f(z)=f(z) .
$$

This implies $\operatorname{Ind}_{K_{\mathbb{C}}(x)}^{Q} \chi$ is completely reducible as a $Q$-module.

By Frobenius reciprocity, $\operatorname{Ind}_{K_{\mathbb{C}}(x)}^{K_{\mathbb{C}}} \chi=\operatorname{Ind}_{Q}^{K_{\mathbb{C}}} \operatorname{Ind}_{K_{\mathbb{C}}(x)}^{Q} \chi$. Assume $\mathcal{O}=K_{\mathbb{C}} \cdot x$ has p-height less than or equal to 2. By Proposition 2.8. $\operatorname{Ind}_{K_{\mathbb{C}}(x)}^{Q} \chi \simeq \operatorname{Ind}_{L_{\mathbb{C}}(x)}^{L_{\mathbb{C}}} \chi$, where $\operatorname{Ind}_{L_{\mathbb{C}}(x)}^{L_{\mathbb{C}}} \chi$ is an $L_{\mathbb{C}}(x)$-module extended to $Q$ by trivial $U$-action. In fact, D. King has obtained $\mathfrak{l}$ and $\mathfrak{l}(x)$ in [10], but $L(x)$ is not necessarily connected even if $L$ is connected. Then we use the Kostant's Borel-Weil theorem ([11, Theorem 6.4, see also [1, Theorem 3.10) to calculate $\operatorname{Ind}_{Q}^{K_{\mathbb{C}}}$.

Finally, we need to determine the character $\chi$. Because of the definition,

$$
\mathrm{d} \gamma \simeq\left(\bigwedge^{\operatorname{dim} \mathfrak{k} / \mathfrak{k}(x)} \mathfrak{k} / \mathfrak{k}(x)\right)^{*} .
$$


With the following proposition, if we determine $\mathfrak{u}^{-}$is an $\mathfrak{l}$-module, we will determine $\mathrm{d} \gamma$ and $\chi$.

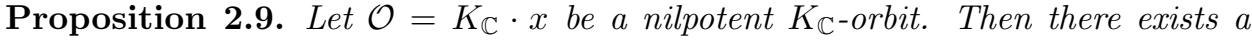
$\mathfrak{k}(x)$-modules isomorphism

$$
\left(\bigwedge^{\operatorname{dim} \mathfrak{k} / \mathfrak{k}(x)} \mathfrak{k} / \mathfrak{k}(x)\right)^{*} \simeq\left(\bigwedge^{\operatorname{dim} \mathfrak{u}^{-}} \mathfrak{u}^{-}\right)^{*} \simeq \bigwedge^{\operatorname{dim} \mathfrak{u}} \mathfrak{u} .
$$

Proof. Since the restriction of $\left.\mathrm{d} \gamma\right|_{\mathfrak{u}(x)}$ is trivial, it's enough to prove that

$$
\left(\bigwedge^{\operatorname{dim} \mathfrak{k} / \mathfrak{k}(x)} \mathfrak{k} / \mathfrak{k}(x)\right)^{*} \simeq\left(\bigwedge^{\operatorname{dim} \mathfrak{u}^{-}} \mathfrak{u}^{-}\right)^{*}
$$

as $\mathfrak{l}(x)$-modules. Moreover, $\mathfrak{l}(x)$ is the direct sum of its semisimple part s.s.l $(x)$ and center $\mathfrak{c}$, i.e.,

$$
\mathfrak{l}(x)=\operatorname{s.s.} \mathfrak{l}(x)+\mathfrak{c} .
$$

Since $\mathrm{d} \gamma$ is one dimensional, $\left.\mathrm{d} \gamma\right|_{\text {s.s.l }(x)}$ is trivial. It's enough to prove that $\left(\bigwedge^{\operatorname{dim} \mathfrak{k} / \mathfrak{k}(x)} \mathfrak{k} / \mathfrak{k}(x)\right)^{*}$ and $\left(\bigwedge^{\operatorname{dim} \mathfrak{u}^{-}} \mathfrak{u}^{-}\right)^{*}$ are isomorphic as $\mathfrak{c}$-modules. Since both $\mathfrak{l}(x)$ and $\mathfrak{l}$ are reductive Lie algebras, there exists a Cartan subalgebra $\mathfrak{t} \subset \mathfrak{l}$, such that $\mathfrak{t} \cap \mathfrak{l}(x)$ is a Cartan subalgebra of $\mathfrak{l}(x)$ and $\mathfrak{c} \subset \mathfrak{t}$. Then $\mathfrak{c}$ acts trivially on

$$
\left(\bigwedge^{\operatorname{dim} \mathfrak{l}} \mathfrak{l}\right)^{*} \text { and }\left(\bigwedge^{\operatorname{dim} \mathfrak{l}(x)} \mathfrak{l}(x)\right)^{*} \text {. }
$$

Moreover $\mathfrak{c}$ acts trivially on

$$
\left(\bigwedge^{\operatorname{dim} \mathfrak{l} / \mathfrak{l}(x)} \mathfrak{l} / \mathfrak{l}(x)\right)^{*}
$$

because

$$
\left(\bigwedge^{\operatorname{dim} \mathfrak{l}} \mathfrak{l}\right)^{*} \simeq\left(\bigwedge^{\operatorname{dim} \mathfrak{l} / \mathfrak{l}(x)} \mathfrak{l} / \mathfrak{l}(x)\right)^{*} \otimes\left(\bigwedge^{\operatorname{dim} \mathfrak{l}(x)} \mathfrak{l}(x)\right)^{*}
$$

Since

$$
\mathfrak{k} / \mathfrak{k}(x) \simeq \mathfrak{l} / \mathfrak{l}(x)+\mathfrak{u}^{-}
$$

as $\mathfrak{l}(x)$-modules, we have

$$
\mathrm{d} \gamma \simeq\left(\bigwedge^{\operatorname{dim} \mathfrak{l} / \mathfrak{l}(x)} \mathfrak{l} / \mathfrak{l}(x)\right)^{*} \otimes\left(\bigwedge^{\operatorname{dim} \mathfrak{u}^{-}} \mathfrak{u}^{-}\right)^{*} \simeq\left(\bigwedge^{\operatorname{dim} \mathfrak{u}^{-}} \mathfrak{u}^{-}\right)^{*} \simeq \bigwedge^{\operatorname{dim} \mathfrak{u}} \mathfrak{u}
$$

\section{Reductive DuAl PAIR AND Theta Lifting}

This section reviews some fundamental results on dual pairs. Specifically it focuses on the type I dual pairs in the stable range.

3.1. Preliminary of theta lifting. We fix an oscillator representation $\Omega$ of $\operatorname{Sp}(2 N, \mathbb{R})$. It is a representation of the metaplectic twofold cover $M p(2 N, \mathbb{R})$ of $S p(2 N, \mathbb{R})$. Let $\mathbb{Z}_{2}=\{ \pm 1\}$ be the kernel of the projection pr : $M p(2 N, \mathbb{R}) \rightarrow$ $S p(2 N, \mathbb{R})$. Let $\left(G, G^{\prime}\right)$ be a reductive dual pair in $S p(2 N, \mathbb{R})$, and $\widetilde{G}$, $\widetilde{G^{\prime}}$ be the corresponding inverse images in $M p(2 N, \mathbb{R})$. Let $\mathcal{C}\left(\mathfrak{g}^{\prime}, \widetilde{K^{\prime}}\right)$ be the set of the isomorphic classes of irreducible $\left(\mathfrak{g}^{\prime}, \widetilde{K^{\prime}}\right)$-modules, and $R\left(\mathfrak{g}^{\prime}, \widetilde{K^{\prime}} ; \Omega\right)$ be the subset of $\mathcal{C}\left(\mathfrak{g}^{\prime}, \widetilde{K^{\prime}}\right)$ which can be realized as a quotient of $\Omega$. Given any $\pi^{\prime} \in R\left(\mathfrak{g}^{\prime}, \widetilde{K^{\prime}} ; \Omega\right)$, let 
$N_{\pi^{\prime}}$ be the intersection of all subspaces $N \subset \Omega$ such that $\Omega / N$ is a $\left(\mathfrak{g}^{\prime}, \widetilde{K^{\prime}}\right)$-module isomorphic to $\pi^{\prime}$. Then $\Omega / N_{\pi^{\prime}}$ is a $\left(\mathfrak{g} \oplus \mathfrak{g}^{\prime}, \widetilde{K} \cdot \widetilde{K^{\prime}}\right)$-module and has decomposition

$$
\Omega / N_{\pi^{\prime}} \simeq \Omega\left(\pi^{\prime}\right) \otimes \pi^{\prime},
$$

where $\Omega\left(\pi^{\prime}\right)$ is a $(\mathfrak{g}, \widetilde{K})$-module. We call $\Omega\left(\pi^{\prime}\right)$ Howe's maximal quotient.

Theorem 3.1 (4], Theorem 2.1). The $(\mathfrak{g}, \widetilde{K})$-module $\Omega\left(\pi^{\prime}\right)$ is finitely generated, admissible, and quasisimple. Furthermore, $\Omega\left(\pi^{\prime}\right)$ has a unique irreducible $(\mathfrak{g}, \widetilde{K})$ quotient $\pi$, and the correspondence $\pi^{\prime} \leftrightarrow \pi$ defines a bijection between $R\left(\mathfrak{g}^{\prime}, \widetilde{K^{\prime}} ; \Omega\right)$ and $R(\mathfrak{g}, \widetilde{K} ; \Omega)$. We denote this map by $\pi=\theta\left(\pi^{\prime}\right)$.

Definition 3.2. We call a unitary representation of $\widetilde{G}$ genuine if its restriction to $\mathbb{Z}_{2}$ is a multiple of the nontrivial character of $\mathbb{Z}_{2}$.

Let $\hat{\widetilde{G}}(\epsilon)$ be the set of irreducible unitary genuine representations of $\widetilde{G}$. Analogous notation is used for other groups. The following theorem, which is proved by J.-S. Li, is a powerful tool in studying the unitarity preservation of theta lifting.

Theorem 3.3 ([14, Theorem A). Suppose $\left(G, G^{\prime}\right)$ is in the stable range with $G^{\prime}$ the smaller member. Then Howe's duality correspondence gives rise to an injection

$$
\hat{\bar{G}^{\prime}}(\epsilon) \rightarrow \hat{\widetilde{G}}(\epsilon) .
$$

Thus in the stable range, duality correspondence preserves unitarity, and the whole set $\hat{\bar{G}^{\prime}}(\epsilon)$ is contained in the domain of the correspondence. (If $\left(G, G^{\prime}\right)=(O(2 n, 2 n)$, $S p(2 n, \mathbb{R}))$, we exclude the trivial representation of $S p(2 n, \mathbb{R})$.)

3.2. Theta lifting of unitary character. For certain dual pairs, we have some method to determine the $K$-types of the theta lifting of the unitary character. Zhu and Huang proved the following theorem.

Theorem 3.4 ([25], Proposition 2.1). Assume the setting of Theorem 3.3. Let $\xi$ be a genuine unitary character of $\widetilde{G^{\prime}}$. Then Howe's maximal quotient $\Omega(\xi)$ is irreducible and unitary.

We consider the following see-saw pair,

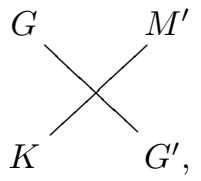

and denote the theta lifting from $K$ to $M^{\prime}$ by $\rho$.

Theorem 3.5 ([17, Proposition 3.3). Let $\left(G, G^{\prime}\right)$ be a dual pair in the stable range with $G^{\prime}$ the smaller member and let $\xi$ be a unitary character of $\widetilde{G^{\prime}}$. The K-types decomposition of Howe's maximal quotient $\Omega(\xi)$ is given by

$$
\left.\Omega(\xi)\right|_{\widetilde{K}} \simeq \sum_{\tau} \operatorname{dim} \operatorname{Hom}_{\widetilde{G^{\prime}}}(\rho(\tau), \xi) \cdot \tau .
$$

Combining these two theorems, we have the following corollary. 
Corollary 3.6. Let $\left(G, G^{\prime}\right)$ and $\xi$ be the same as in Theorem 3.4. Then the K-types decomposition of $\theta(\xi)$ is given by

$$
\left.\theta(\xi)\right|_{\widetilde{K}} \simeq \sum_{\tau} \operatorname{dim} \operatorname{Hom}_{\widetilde{G^{\prime}}}(\rho(\tau), \xi) \cdot \tau
$$

\section{QuAntization of SPherical NilPotent orbits}

This section consists of three parts. The first is the computation of the spherical admissible orbits of $S p(2 n, \mathbb{R})(M p(2 n, \mathbb{R}))$ and the determination on the global sections $\operatorname{Ind}_{K_{\mathbb{C}}(x)}^{K_{\mathbb{C}}} \chi \otimes \tau_{n}^{\frac{q-p}{2}} \mathbf{1}_{n}$ of the line bundle associated to the admissible datum $\left(x, \chi \otimes \tau_{n}^{\frac{q-p}{2}} \mathbf{1}_{n}\right)$. The second part is the computation of the $K$-types of the representation $\theta(\xi)$ obtained by theta lifting of a unitary character of $\widetilde{O(p, q)}$. The last part derives the unitary $(\mathfrak{g}, K)$-module structure on $\operatorname{Ind}_{K_{\mathbb{C}}(x)}^{K_{\mathbb{C}}} \chi \otimes \tau_{n}^{\frac{q-p}{2} \mathbf{1}_{n}}$ by comparing the $K$-types of $\theta(\xi)^{*}$ with $\operatorname{Ind}_{K_{\mathbb{C}}(x)}^{K_{\mathbb{C}}} \chi \otimes \tau_{n}^{\frac{q-p}{2}} \mathbf{1}_{n}$.

4.1. Nilpotent orbits of $\mathfrak{s p}(2 n, \mathbb{R})$. The real symplectic group is the subgroup of $G L(2 n, \mathbb{R})$ which leaves the symplectic form invariant given by

$$
\left[\begin{array}{cc}
0 & I_{n} \\
-I_{n} & 0
\end{array}\right]
$$

where $I_{n}$ is the identity matrix of order $n$. The Lie algebra of $S p(2 n, \mathbb{R})$ is

$$
\mathfrak{s p}(2 n, \mathbb{R})=\left\{\left[\begin{array}{cc}
A & B \\
C & -A^{t}
\end{array}\right] \mid A, B, C \text { real } n \times n \text { matrices; } B, C \text { symmetric }\right\} .
$$

The complexification of $\mathfrak{s p}(2 n, \mathbb{R})$ is $\mathfrak{s p}(2 n, \mathbb{C})$, and the complexified Cartan involution is given by

$$
\theta\left(\left[\begin{array}{cc}
A & B \\
C & -A^{t}
\end{array}\right]\right)=\left[\begin{array}{cc}
-A^{t} & -C^{t} \\
-B^{t} & A
\end{array}\right], \forall\left[\begin{array}{cc}
A & B \\
C & -A^{t}
\end{array}\right] \in \mathfrak{s p}(2 n, \mathbb{C}) .
$$

The conjugation $\sigma$ with respect to the real form $\mathfrak{s p}(2 n, \mathbb{R})$ is the ordinary complex conjugation.

We define

$$
I_{p,-q}^{n}=\left[\begin{array}{ccc}
I_{p} & 0 & 0 \\
0 & 0 & 0 \\
0 & 0 & -I_{q}
\end{array}\right]_{n \times n}, I_{p, q}^{n}=\left[\begin{array}{ccc}
I_{p} & 0 & 0 \\
0 & 0 & 0 \\
0 & 0 & I_{q}
\end{array}\right]_{n \times n},
$$

and put

$x=\frac{1}{2}\left[\begin{array}{cc}I_{p, q}^{n} & i I_{p,-q}^{n} \\ i I_{p,-q}^{n} & -I_{p, q}^{n}\end{array}\right], h=\left[\begin{array}{cc}0 & -i I_{p,-q}^{n} \\ i I_{p,-q}^{n} & 0\end{array}\right], y=\frac{1}{2}\left[\begin{array}{cc}I_{p, q}^{n} & -i I_{p,-q}^{n} \\ -i I_{p,-q}^{n} & -I_{p, q}^{n}\end{array}\right]$.

It follows immediately that $\{x, h, y\}$ is a Kostant-Sekiguchi triple of $S p(2 n, \mathbb{R})$ corresponding to the signed Young diagram $(+2)^{p}(-2)^{q}(+1)^{2(n-p-q)}$.

The maximal compact subgroup $K$ of $S p(2 n, \mathbb{R})$ is isomorphic to $U(n)$, and the embedding is given by

$$
\iota: U(n) \rightarrow S p(2 n, \mathbb{R}), \iota(A+B i)=\left[\begin{array}{cc}
A & B \\
-B & A
\end{array}\right] .
$$

Since $h=-i \iota\left(i I_{p,-q}^{n}\right)$, the centralizer of $h$ is isomorphic to

$$
L \simeq U(p) \times U(n-p-q) \times U(q) .
$$


We put

$$
g=\iota\left(\left[\begin{array}{ccc}
A_{11}+i B_{11} & 0 & 0 \\
0 & A_{22}+i B_{22} & 0 \\
0 & 0 & A_{33}+i B_{33}
\end{array}\right]\right) \in K .
$$

The equality $g x=x g$ means

$$
\begin{aligned}
& {\left[\begin{array}{cccccc}
A_{11}+i B_{11} & 0 & 0 & i A_{11}-B_{11} & 0 & 0 \\
0 & 0 & 0 & 0 & 0 & 0 \\
0 & 0 & A_{33}-i B_{33} & 0 & 0 & -i A_{33}-B_{33} \\
i A_{11}-B_{11} & 0 & 0 & -A_{11}-i B_{11} & 0 & 0 \\
0 & 0 & 0 & 0 & 0 & 0 \\
0 & 0 & -i A_{33}-B_{33} & 0 & 0 & -A_{33}+i B_{33}
\end{array}\right]} \\
& =\left[\begin{array}{cccccc}
A_{11}-i B_{11} & 0 & 0 & i A_{11}+B_{11} & 0 & 0 \\
0 & 0 & 0 & 0 & 0 & 0 \\
0 & 0 & A_{33}+i B_{33} & 0 & 0 & -i A_{33}+B_{33} \\
i A_{11}+B_{11} & 0 & 0 & -A_{11}+i B_{11} & 0 & 0 \\
0 & 0 & 0 & 0 & 0 & 0 \\
0 & 0 & -i A_{33}+B_{33} & 0 & 0 & -A_{33}-i B_{33}
\end{array}\right] .
\end{aligned}
$$

This implies $B_{11}=0, B_{33}=0$ and

$$
L(x) \simeq O(p) \times U(n-p-q) \times O(q) .
$$

Let $\widetilde{G}$ be the metaplectic twofold cover $M p(2 n, \mathbb{R})$. Then there exists a nonsplit exact sequence

$$
1 \rightarrow \mathbb{Z}_{2} \rightarrow \widetilde{G} \stackrel{\text { pr }}{\rightarrow} G \rightarrow 1
$$

Let $H$ be a subgroup of $S p(2 n, \mathbb{R})$ and $\widetilde{H}$ be the inverse image of $H$ in $\widetilde{G}$. The projection pr induces an isomorphism between the Lie algebra of $\widetilde{H}$ and the Lie algebra of $H$. Without confusion, we write $\mathfrak{h}_{0}$ for the Lie algebra of $\widetilde{H}$. Since the image of $\mathbb{Z}_{2}$ is contained in the center of $M p(2 n, \mathbb{R})$, we can parameterize the coadjoint orbits of $M p(2 n, \mathbb{R})$ by the coadjoint orbits of $S p(2 n, \mathbb{R})$. Then the spher-

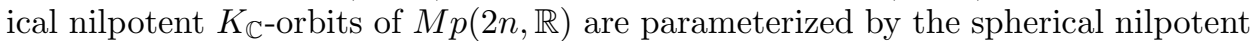

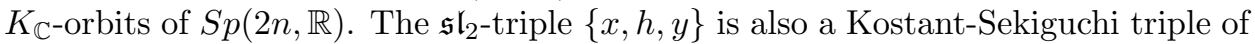
$M p(2 n, \mathbb{R})$ corresponding to the signed Young diagram $(+2)^{p}(-2)^{q}(+1)^{2(n-p-q)}$, and the centralizer of $h$ in $\widetilde{K}$ is isomorphic to $\widetilde{L}$.

By Proposition 4.5 in [23, we have the following proposition.

Proposition 4.1. Let $\mathcal{O}$ be a nilpotent orbit corresponding to the signed Young diagram $(+2)^{p}(-2)^{q}(+1)^{2(n-p-q)}$. Then it is a Vogan orbit if and only if $n>$ $p+q$. Let $\{x, h, y\}$ be a Kostant-Sekiguchi triple corresponding to $\mathcal{O}$, and $Q=L_{\mathbb{C}} U$ (resp. $\left.\widetilde{Q}=\widetilde{L}_{\mathbb{C}} U\right)$ be the parabolic subgroup of $K_{\mathbb{C}}\left(\right.$ resp. $\left.\widetilde{K}_{\mathbb{C}}\right)$ defined by ad $h$. Then

$$
L_{\mathbb{C}} \simeq G L(p, \mathbb{C}) \times G L(n-p-q, \mathbb{C}) \times G L(q, \mathbb{C}), \widetilde{L}_{\mathbb{C}} \simeq \widetilde{L_{\mathbb{C}}}
$$

Let $K_{\mathbb{C}}(x)$ (resp. $\left.\widetilde{K}_{\mathbb{C}}(x)\right)$ be the stabilizer of $x$. We have Levi decomposition $K_{\mathbb{C}}(x)$ $=L_{\mathbb{C}}(x) U\left(\operatorname{resp} . \widetilde{K}_{\mathbb{C}}(x)=\widetilde{L}_{\mathbb{C}}(x) U\right)$ with

$$
L_{\mathbb{C}}(x) \simeq O(p, \mathbb{C}) \times G L(n-p-q, \mathbb{C}) \times O(q, \mathbb{C}) \quad\left(\text { resp. } \widetilde{L}_{\mathbb{C}}(x)=\widetilde{L_{\mathbb{C}}(x)}\right) .
$$


Proof. The complexification of $\mathcal{O}$ is a nilpotent orbit in $\mathfrak{s p}(2 n, \mathbb{C})$ corresponding to the Young diagram $2^{p+q} 1^{2(n-p-q)}$. By Theorem 6.1.3 in [3], we have

$$
\operatorname{dim}_{\mathbb{C}} \mathcal{O}=\frac{1}{2}(p+q)(2 n-p-q+1) .
$$

Then

$$
\operatorname{dim}_{\mathbb{C}} \mathcal{O}-\operatorname{dim}_{\mathbb{C}}(\overline{\mathcal{O}}-\mathcal{O})=n-p-q+1 .
$$

It's a Vogan orbit if and only if $p+q<n$. Because $L_{\mathbb{C}}\left(\operatorname{resp} . L_{\mathbb{C}}(x)\right)$ is the complexification of $L$ (resp. $L(x))$,

$$
L_{\mathbb{C}} \simeq G L(p, \mathbb{C}) \times G L(n-p-q, \mathbb{C}) \times G L(q, \mathbb{C})
$$

and

$$
L_{\mathbb{C}}(x) \simeq O(p, \mathbb{C}) \times G L(n-p-q, \mathbb{C}) \times O(q, \mathbb{C}) .
$$

Since $\widetilde{L}(\operatorname{resp} . \widetilde{L}(x))$ is the twofold cover of $L(\operatorname{resp} . L(x)), \widetilde{L}_{\mathbb{C}} \simeq \widetilde{L_{\mathbb{C}}}\left(\operatorname{resp} . \widetilde{L}_{\mathbb{C}}(x) \simeq\right.$ $\left.\widehat{L_{\mathbb{C}}(x)}\right)$.

4.2. Line bundle associated to the admissible datum. First, we consider $S p(2 n, \mathbb{R})$. Since $\mathfrak{l}(x) \simeq \mathfrak{s o}(p, \mathbb{C}) \times \mathfrak{s l}(n-p-q, \mathbb{C}) \times \mathfrak{s o}(q, \mathbb{C})+\mathbb{C} c$ with

$$
c=\left[\begin{array}{ccc}
0 & 0 & 0 \\
0 & I_{n-p-q} & 0 \\
0 & 0 & 0
\end{array}\right]
$$

and $\mathrm{d} \gamma$ is trivial on $\mathfrak{s o}(p, \mathbb{C}) \times \mathfrak{s l}(n-p-q, \mathbb{C}) \times \mathfrak{s o}(q, \mathbb{C})$, it's enough to determine the action of $c$ on $\mathfrak{u}^{-}$. Furthermore, $c$ is contained in a Cartan subalgebra of $\mathfrak{l}$. In the following table, we list the weights of $\mathfrak{u}^{-}$as $\mathfrak{l}$-module:

TABLE 1. Weights of $\mathfrak{u}^{-}$as $\mathfrak{l}$-module

\begin{tabular}{|c|c|}
\hline$-e_{i}+e_{j}$ & $1 \leq i \leq p, p+1 \leq j \leq n-q$ \\
\hline$-e_{i}+e_{j}$ & $1 \leq i \leq p, n-q+1 \leq j \leq n$ \\
\hline$-e_{i}+e_{j}$ & $p+1 \leq i \leq n-q, n-q+1 \leq j \leq n$ \\
\hline
\end{tabular}

By Proposition 2.9 ,

$$
\mathrm{d} \gamma \simeq 1 \otimes \tau_{n-p-q}^{(q-p) \mathbf{1}_{n-p-q}} \otimes 1,
$$

where $\tau_{n-p-q}^{(q-p) \mathbf{1}_{n-p-q}}$ is an irreducible finite dimensional highest weight $\mathfrak{g l}(n-p-q, \mathbb{C})$ module with highest weight

$$
(q-p) \mathbf{1}_{n-p-q}=(q-p)(\underbrace{1, \ldots, 1}_{n-p-q \text { times }}) .
$$

Then $\frac{1}{2} \mathrm{~d} \gamma$ is a character of $K_{\mathbb{C}}(x)$ if and only if $p+q$ is even. In this case, $\tau_{n}^{\frac{q-p}{2}} \mathbf{1}_{n}$ is a character of $K_{\mathbb{C}}$, and $\left.\tau_{n}^{\frac{q-p}{2}} \mathbf{1}_{n}\right|_{K_{\mathbb{C}}(x)} \simeq \frac{1}{2} \mathrm{~d} \gamma$. Then we have the following proposition.

Proposition 4.2. The spherical Vogan orbit $(+2)^{p}(-2)^{q}(+1)^{2(n-p-q)}$ of $S p(2 n, \mathbb{R})$ is admissible if and only if $p+q$ is even. If $p q \neq 0$, the admissible data $\{(x, \chi \otimes$ $\left.\left.\tau_{n}^{\frac{q-p}{2} \mathbf{1}_{n}}\right)\right\}$ are given by $\left.\left(\chi \otimes \tau_{n}^{\frac{q-p}{2}} \mathbf{1}_{n}\right)\right|_{L_{\mathbb{C}}(x)}$, where $\chi$ is one of following:

$\operatorname{det} \otimes 1 \otimes \operatorname{det}, 1 \otimes 1 \otimes 1$,

$1 \otimes 1 \otimes \operatorname{det}, \operatorname{det} \otimes 1 \otimes 1$.

If $p q=0$, only the first two terms are left. 
Now we turn to the metaplectic twofold cover $M p(2 n, \mathbb{R}) . \widetilde{K}$ is a nonsplit twofold cover of $U(n)$, and $\tau_{n}^{\frac{q-p}{2}} \mathbf{1}_{n}$ is a character of $\widetilde{K}$ and then $\widetilde{K}_{\mathbb{C}}$. The twofold cover $\widetilde{K}$ can be realized as

$$
\widetilde{K}=\left\{(g, z) \in U(n) \times \mathbb{C} \mid \operatorname{det} g=z^{2}\right\} .
$$

As

$$
\mathfrak{l}(x) \simeq \mathfrak{s o}(p, \mathbb{C}) \times \mathfrak{g l}(n-p-q, \mathbb{C}) \times \mathfrak{s o}(q, \mathbb{C}),
$$

we have

$$
\left.\tau_{n}^{\frac{q-p}{2}} \mathbf{1}_{n}\right|_{\mathfrak{l}(x)} \simeq 1 \otimes \tau_{n-p-q}^{\frac{q-p}{2}} \mathbf{1}_{n-p-q} \bigotimes 1
$$

Since $\tau_{n}^{\frac{q-p}{2}} \mathbf{1}_{n}$ is a character of $\widetilde{K}_{\mathbb{C}}(x)$ such that $\left.\tau_{n}^{\frac{q-p}{2}} \mathbf{1}_{n}\right|_{\mathfrak{k}(x)} \simeq \frac{1}{2} \mathrm{~d} \gamma$, we can parameterize the admissible data by the representations of $K_{\mathbb{C}}(x)$ (or more precisely $\left.L_{\mathbb{C}}(x)\right)$.

Proposition 4.3. The spherical Vogan orbit $(+2)^{p}(-2)^{q}(+1)^{2(n-p-q)}$ of $M p(2 n, \mathbb{R})$ is admissible for all $p, q$. If $p q \neq 0$, the admissible data $\left\{\left(x, \chi \otimes \tau_{n}^{\frac{q-p}{2}} \mathbf{1}_{n}\right)\right\}$ are $p a-$ rameterized by $\left.\chi\right|_{L_{\mathbb{C}}(x)}$, where $\chi$ is given by

$\operatorname{det} \otimes 1 \otimes \operatorname{det}, 1 \otimes 1 \otimes 1$,

$1 \otimes 1 \otimes \operatorname{det}, \operatorname{det} \otimes 1 \otimes 1$.

If $p q=0$, only the first two terms are left.

Moreover, the $K$-types of the space of algebraic global sections are given by the following theorem.

Theorem 4.4. Let $\mathcal{O}$ be an admissible Vogan orbit of $\operatorname{Sp}(2 n, \mathbb{R})(\operatorname{resp} . M p(2 n, \mathbb{R}))$ corresponding to the signed Young diagram $(+2)^{p}(-2)^{q}(+1)^{2(n-p-q)}$. If $p q \neq 0$, the spaces of algebraic global sections of the line bundle associated to admissible data are given by

$$
\sum_{\substack{a_{i} \text { are odd, } b_{\text {i }} \text { are odd, } \\ a_{1} \geq \cdots \geq a_{p} \geq 0 \geq-b_{1} \geq \cdots \geq-b_{q}}} \tau_{n}^{\frac{q-p}{2} \mathbf{1}_{n}+\left[b_{q}, \ldots, b_{1}, \mathbf{0}_{n-p-q},-a_{p}, \ldots,-a_{1}\right]},
$$

$$
\begin{aligned}
& \sum_{n^{\frac{q-p}{2}} \mathbf{1}_{n}+\left[b_{q}, \ldots, b_{1}, \mathbf{0}_{n-p-q},-a_{p}, \ldots,-a_{1}\right],} \\
& a_{i} \text { are even, } b_{i} \text { are even, } \\
& a_{1} \geq \cdots \geq a_{p} \geq 0 \geq-b_{1} \geq \cdots \geq-b_{q} \\
& \sum_{\substack{a_{i} \text { are odd, } b_{i} \text { are even, } \\
a_{1} \geq \cdots \geq a_{p} \geq 0 \geq-b_{1} \geq \cdots \geq-b_{q}}} \tau_{n}^{\frac{q-p}{2} \mathbf{1}_{n}+\left[b_{q}, \ldots, b_{1}, \mathbf{0}_{n-p-q},-a_{p}, \ldots,-a_{1}\right]}, \\
& \sum_{\substack{a_{i} \text { are even, } b_{i} \text { are odd, } \\
a_{1} \geq \cdots \geq a_{p} \geq 0 \geq-b_{1} \geq \cdots \geq-b_{q}}} \tau_{n}^{\frac{q-p}{2} \mathbf{1}_{n}+\left[b_{q}, \ldots, b_{1}, \mathbf{0}_{n-p-q},-a_{p}, \ldots,-a_{1}\right]} .
\end{aligned}
$$


Each corresponds to a line bundle which is associated to an admissible datum given in Proposition 4.2 (resp. Proposition 4.3). If $p q=0$, only the first two terms are left.

Proof. We prove this theorem for $S p(2 n, \mathbb{R})$ and the computations for $M p(2 n, \mathbb{R})$ are left to the reader. By Frobenius reciprocity,

$$
\begin{aligned}
\operatorname{Ind}_{K_{\mathbb{C}}(x)}^{K_{\mathbb{C}}}\left(\chi \otimes \tau_{n}^{\frac{q-p}{2}} \mathbf{1}_{n}\right) & =\sum \operatorname{dim} \operatorname{Hom}_{K_{\mathbb{C}}(x)}\left(\tau, \chi \otimes \tau_{n}^{\frac{q-p}{2}} \mathbf{1}_{n}\right) \cdot \tau \\
& =\sum \operatorname{dim} \operatorname{Hom}_{K_{\mathbb{C}}(x)}\left(\tau \otimes \tau_{n}^{\frac{p-q}{2}} \mathbf{1}_{n}, \chi\right) \cdot \tau \\
& =\sum \operatorname{dim} \operatorname{Hom}_{K_{\mathbb{C}}(x)}\left(\tau \otimes \tau_{n}^{\frac{p-q}{2}} \mathbf{1}_{n}, \chi\right) \cdot\left(\tau \otimes \tau_{n}^{\frac{p-q}{2}} \mathbf{1}_{n}\right) \otimes \tau_{n}^{\frac{q-p}{2}} \mathbf{1}_{n} \\
& =\tau_{n}^{\frac{q-p}{2}} \mathbf{1}_{n} \otimes \operatorname{Ind}_{K_{\mathbb{C}}(x)}^{K_{\mathbb{C}}} \chi .
\end{aligned}
$$

In order to calculate $\operatorname{Ind}_{K_{\mathbb{C}}(x)}^{K_{\mathbb{C}}} \chi$, we calculate $\operatorname{Ind}_{K_{\mathbb{C}}(x)}^{Q} \chi$ first. It is clear that the irreducible representation of $Q$ is given by

$$
\tau_{p}^{\alpha} \otimes \tau_{n-p-q}^{\gamma} \otimes \tau_{q}^{\beta},
$$

where $\alpha, \gamma, \beta$ are the highest weights of the corresponding irreducible finite dimensional representations. We only consider the case of $\chi \simeq 1 \otimes 1 \otimes 1$. Others are similar.

After calculating the eigenvalue of ad $h$ on $\mathfrak{s p}(2 n, \mathbb{R})$, it follows immediately that this orbit has $\mathfrak{g}$-height 2. By Proposition 2.8 $\operatorname{Ind}_{K_{\mathbb{C}}(x)}^{Q} \chi$ is completely reducible and

$$
\operatorname{Ind}_{K_{\mathbb{C}}(x)}^{Q} \chi=\sum \operatorname{dim} \operatorname{Hom}_{K_{\mathbb{C}}(x)}\left(\tau_{p}^{\alpha} \otimes \tau_{n-p-q}^{\gamma} \otimes \tau_{q}^{\beta}, \chi\right) \cdot \tau_{p}^{\alpha} \otimes \tau_{n-p-q}^{\gamma} \otimes \tau_{q}^{\beta} .
$$

Because the $U$-action is trivial,

$$
\begin{aligned}
\operatorname{Hom}_{K_{\mathbb{C}}(x)}\left(\tau_{p}^{\alpha} \otimes \tau_{n-p-q}^{\gamma} \otimes \tau_{q}^{\beta}, \chi\right) & =\operatorname{Hom}_{L_{\mathbb{C}}(x)}\left(\tau_{p}^{\alpha} \otimes \tau_{n-p-q}^{\gamma} \otimes \tau_{q}^{\beta}, \chi\right) \\
& =\operatorname{Hom}_{L_{\mathbb{C}}(x)}\left(\tau_{p}^{\alpha} \otimes \tau_{n-p-q}^{\gamma} \otimes \tau_{q}^{\beta}, 1 \otimes 1 \otimes 1\right) .
\end{aligned}
$$

Then $\operatorname{dim} \operatorname{Hom}_{K_{\mathbb{C}}(x)}\left(\tau_{p}^{\alpha} \otimes \tau_{n-p-q}^{\gamma} \otimes \tau_{q}^{\beta}, \chi\right) \neq 0$ if and only if

$$
\alpha, \beta \text { are even integers, } \tau_{n-p-q}^{\gamma} \simeq 1 \text {. }
$$

Let $\alpha=\left[a_{1}, \ldots, a_{p}\right], \beta=\left[b_{1}, \ldots, b_{q}\right]$. The lowest weight of

$$
\tau_{p}^{\alpha} \otimes 1 \otimes \tau_{q}^{\beta}
$$

is given by

$$
\left[-\alpha^{*}, \mathbf{0}_{n-p-q},-\beta^{*}\right]
$$

where $\alpha^{*}\left(\operatorname{resp} . \beta^{*}\right)$ is the highest weight of $\left(\tau_{p}^{\alpha}\right)^{*}\left(\operatorname{resp} .\left(\tau_{q}^{\beta}\right)^{*}\right)$. By Kostant's Borel-Weil theorem,

$$
\operatorname{Ind}_{Q}^{K_{\mathbb{C}}} \tau_{p}^{\alpha} \otimes 1 \otimes \tau_{q}^{\beta} \neq 0
$$

if and only if

$$
a_{p} \leq \cdots \leq a_{1} \leq 0 \leq b_{q} \leq \cdots \leq b_{1}
$$

Then

$$
\operatorname{Ind}_{K_{\mathbb{C}}(x)}^{K_{\mathbb{C}}} \chi=\sum \tau_{n}^{\left[\beta, \mathbf{0}_{n-p-q}, \alpha\right]}
$$


where $\alpha=\left[a_{1}, \ldots, a_{p}\right], \beta=\left[b_{1}, \ldots, b_{q}\right], a_{p} \leq \cdots \leq a_{1} \leq 0 \leq b_{q} \leq \cdots \leq b_{1}$, and all $a_{i}, b_{j}$ are even. With a slight adjustment to our notation, then

$$
\begin{aligned}
& \operatorname{Ind}_{K_{\mathbb{C}}(x)}^{K_{\mathbb{C}}}\left(\chi \otimes \tau_{n}^{\frac{q-p}{2}} \mathbf{1}_{n}\right) \\
& \simeq \sum_{\substack{a_{i} \text { are even, } b_{i} \text { are even, } \\
a_{1} \geq \cdots \geq a_{p} \geq 0 \geq-b_{1} \geq \cdots \geq-b_{q}}} \tau_{n}^{\frac{q-p}{2} \mathbf{1}_{n}+\left[b_{q}, \ldots, b_{1}, \mathbf{0}_{n-p-q},-a_{p}, \ldots,-a_{1}\right]} .
\end{aligned}
$$

4.3. Quantization of spherical nilpotent orbits. Let $(O(p, q), S p(2 n, \mathbb{R}))$ be a dual pair in stable range with $O(p, q)$ the smaller member. Let $\xi$ be the unitary character of $\widetilde{O(p, q)}$. We denote the theta lifting of $\xi$ to $\widehat{S p(2 n, \mathbb{R})}$ by $\theta(\xi)$, and the maximal quotient by $\Omega(\xi)$. There exists a see-saw pair

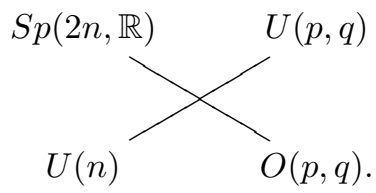

By Corollary 3.6, the $U(n)$-types of $\theta(\xi)$ are given by

$$
\left.\theta(\xi)\right|_{\widetilde{U(n)}}=\sum \operatorname{dim} \operatorname{Hom} \underset{O(p, q)}{ }(\rho(\tau), \xi) \cdot \tau .
$$

Since $\widetilde{U(p, q)}$ is defined by a character $\operatorname{det}^{\frac{n}{2}}$,

$$
\operatorname{dim} \operatorname{Hom}_{\widetilde{O(p, q)}}(\rho(\tau), \xi)=\operatorname{dim} \operatorname{Hom}_{O(p, q)}\left(\rho(\tau) \otimes \operatorname{det}^{-\frac{n}{2}}, \xi \otimes \operatorname{det}^{-\frac{n}{2}}\right) .
$$

We denote $\rho(\tau) \otimes \operatorname{det}^{-\frac{n}{2}}$ by $\bar{\rho}(\tau)$, and $\xi \otimes \operatorname{det}^{-\frac{n}{2}}$ by $\bar{\xi}$. Let $\mathfrak{g}=\mathfrak{k}+\mathfrak{p}$ be the complexified Cartan decomposition of $U(p, q)$. Then $\mathfrak{p}=\mathfrak{p}^{+}+\mathfrak{p}^{-}$. Let $\bar{\sigma}$ be the harmonic $U(p) \times U(q)$-type of $\bar{\rho}(\tau)$. Then $\bar{\rho}(\tau)=\mathcal{U}\left(\mathfrak{p}^{+}\right) \cdot \bar{\sigma}$. Let $\mathfrak{g}^{\prime}=\mathfrak{k}^{\prime}+\mathfrak{p}^{\prime}$ be the complexified Cartan decomposition of $O(p, q)$. Then $\mathfrak{p}^{\prime} \subset \mathfrak{p}$ and $\mathfrak{p}^{+}+\mathfrak{p}^{-}=\mathfrak{p}^{\prime}+\mathfrak{p}^{+}=\mathfrak{p}^{\prime}+\mathfrak{p}^{-}$. This implies $\bar{\rho}(\tau)=\mathcal{U}\left(\mathfrak{p}^{\prime}\right) \cdot \bar{\sigma}$. Let $f$ be a nontrivial homomorphism in $\operatorname{Hom}_{O(p, q)}(\bar{\rho}(\tau), \bar{\xi})$. If the restriction $\left.f\right|_{\bar{\sigma}}$ is trivial, it follows directly that

$$
f(\bar{\rho}(\tau))=f\left(\mathcal{U}\left(\mathfrak{p}^{\prime+}\right) \cdot \bar{\sigma}\right)=\mathcal{U}\left(\mathfrak{p}^{\prime+}\right) \cdot f(\bar{\sigma})=0 .
$$

Then the restriction

$$
\text { Res : } \operatorname{Hom}_{O(p, q)}(\bar{\rho}(\tau), \bar{\xi}) \rightarrow \operatorname{Hom}_{O(p) \times O(q)}(\bar{\sigma}, \bar{\xi}),\left.f \mapsto f\right|_{\bar{\sigma}}
$$

is injective. So

$$
\operatorname{dim} \operatorname{Hom}_{O(p, q)}(\bar{\rho}(\tau), \bar{\xi}) \leq \operatorname{dim} \operatorname{Hom}_{O(p) \times O(q)}(\bar{\sigma}, \bar{\xi}) .
$$

Moreover, $(S O(p) \times S O(q), U(p) \times U(q))$ is a symmetric pair, then

$$
\begin{aligned}
\operatorname{dim} \operatorname{Hom}_{O(p) \times O(q)}(\bar{\sigma}, \bar{\xi}) & \leq \operatorname{dim}_{\operatorname{Hom}} \operatorname{dom}_{(p) \times S O(q)}(\bar{\sigma}, \bar{\xi}) \\
& =\operatorname{dim}_{\operatorname{Hom}}(\bar{\sigma}) \times S O(q) \\
& (\bar{\sigma}, 1) \leq 1 .
\end{aligned}
$$

Summarizing, we have

$$
\operatorname{dim} \operatorname{Hom}_{O(p, q)}(\bar{\rho}(\tau), \bar{\xi}) \leq \operatorname{dim} \operatorname{Hom}_{O(p) \times O(q)}(\bar{\sigma}, \bar{\xi}) \leq 1 .
$$

Kudla and Rallis obtained these results in [12. We parameterize the genuine unitary character of $\widetilde{O(p, q)}$ by the unitary character of $O(p, q)$. The trivial representation and the determinant representation are unitary characters of $O(p, q)$. 
If $p q \neq 0$, there exist $\bar{\xi}_{+,-}$and $\bar{\xi}_{-,+}$such that $\left.\bar{\xi}_{+,-}\right|_{O(p) \times O(q)} \simeq 1 \otimes$ det, and $\bar{\xi}_{-,+} \mid O(p) \times O(q) \simeq \operatorname{det} \otimes 1$. Twisted by a character of $O(p, q)$, the following theorem describes the possible range of the $\widetilde{U(n)}$-types of $\theta(\xi)$.

Theorem 4.5 ([5], Theorem 2.3). Let $\tau$ be a $\widetilde{U(n)}$-type of $\theta(\xi)$, then the highest weight of $\tau$ is of the form

$$
\frac{p-q}{2} \mathbf{1}_{n}+\left[a_{1}, \ldots, a_{p}, \mathbf{0}_{n-p-q},-b_{1}, \ldots,-b_{q}\right],
$$

where $a_{1} \geq \cdots \geq a_{p} \geq 0 \geq-b_{1} \geq \cdots \geq-b_{q}$ are integers. Furthermore, all $a_{i}$ are even if $\left.\bar{\xi}\right|_{O(p)} \simeq 1$, and odd if $\left.\bar{\xi}\right|_{O(p)} \simeq$ det. Similarly all $b_{j}$ are even if $\left.\bar{\xi}\right|_{O(q)} \simeq 1$, and odd if $\left.\bar{\xi}\right|_{O(q)} \simeq$ det.

Given $\tau$ in the possible range, we now explain our method for calculating $\operatorname{dim} \operatorname{Hom}_{O(p, q)}(\bar{\rho}(\tau), \bar{\xi})$. If $\bar{\rho}(\tau)$ occurs in the discrete part of the decomposition

$$
\mathrm{L}^{2}\left(U(p, q) / S O_{e}(p, q)\right) \simeq \int_{\widehat{U(p, q)}}^{\oplus} m_{\pi} \pi \mathrm{d} \mu(\pi),
$$

then $\operatorname{dim} \operatorname{Hom}_{S O_{e}(p, q)}(\bar{\rho}(\tau), 1) \neq 0$. Given any nontrivial $f \in \operatorname{Hom}_{S O_{e}(p, q)}(\bar{\rho}(\tau), 1)$, it can be extended to a homomorphism in $\operatorname{Hom}_{O(p, q)}\left(\bar{\rho}(\tau), \xi^{\prime}\right)$ for some unitary character $\xi^{\prime}$. Since

$$
1 \leq \operatorname{dim} \operatorname{Hom}_{O(p, q)}\left(\bar{\rho}(\tau), \xi^{\prime}\right) \leq \operatorname{dim} \operatorname{Hom}_{O(p) \times O(q)}\left(\bar{\sigma}, \xi^{\prime}\right) \leq 1,
$$

we have $\operatorname{Hom}_{O(p) \times O(q)}\left(\bar{\sigma}, \xi^{\prime}\right)=1$, and $\xi^{\prime}$ is determined by the branching $\operatorname{Res}_{O(p) \times O(q)}^{U(p) \times U(q)} \bar{\sigma}$. Clearly

$$
\operatorname{dim} \operatorname{Hom}_{O(p, q)}(\rho(\tau), \bar{\xi})= \begin{cases}1, & \bar{\xi} \simeq \xi^{\prime} \\ 0, & \text { otherwise }\end{cases}
$$

Theorem 4.6. Assume the setting of Theorem 4.5 and $n \geq p+q$. Then the $\widetilde{U(n)}$-types of $\theta(\xi)$ are given by

$$
\frac{p-q}{2} \mathbf{1}_{n}+\left[a_{1}, \ldots, a_{p}, \mathbf{0}_{n-p-q},-b_{1}, \ldots,-b_{q}\right],
$$

where $a_{1} \geq \cdots \geq a_{p} \geq 0 \geq-b_{1} \geq \cdots \geq-b_{q}$ are integers. Furthermore, all $a_{i}$ are even if $\left.\bar{\xi}\right|_{O(p)} \simeq 1$, and odd if $\left.\bar{\xi}\right|_{O(p)} \simeq$ det. Similarly all $b_{j}$ are even if $\left.\bar{\xi}\right|_{O(q)} \simeq 1$, and odd if $\left.\bar{\xi}\right|_{O(q)} \simeq$ det.

Proof. We twist $\bar{\xi}$ by the character $\hat{\xi}$, where

$$
\hat{\xi} \simeq \begin{cases}1 \otimes \operatorname{det}, & \text { if } n \text { is odd; } \\ 1 \otimes 1, & \text { if } n \text { is even. }\end{cases}
$$

We prove this theorem for $\bar{\xi}_{+,+} \mid O(p) \times O(q) \simeq 1 \otimes 1$; others are left to the reader. Let $\tau$ be a $K$-type in the possible range with highest weight

$$
\frac{p-q}{2} \mathbf{1}_{n}+\left[a_{1}, \ldots, a_{p}, \mathbf{0}_{n-p-q},-b_{1}, \ldots,-b_{q}\right],
$$

where all $a_{i}$ and $b_{j}$ are positive even integers. Then $\left[a_{1}, \ldots, a_{p},-b_{1}-n, \ldots,-b_{q}-n\right]$ is the highest weight of the lowest degree $U(p) \times U(q)$-type of $\bar{\rho}(\tau)$, and $\left[a_{p}, \ldots, a_{1},-b_{q}-\right.$ $\left.n, \ldots,-b_{1}-n\right]$ is the lowest weight of $\bar{\rho}(\tau)$. Furthermore, the infinitesimal character of $\bar{\rho}(\tau)$ is

$$
\left[a_{p}, \ldots, a_{1},-b_{q}-n, \ldots,-b_{1}-n\right]-\rho_{\mathfrak{g}}
$$


where

$$
\rho_{\mathfrak{g}}=\left[\frac{p+q-1}{2}, \frac{p+q-3}{2}, \ldots,-\frac{p+q-1}{2}\right],
$$

and is conjugate to

$\lambda=\left[a_{1}-\frac{-p+q+1}{2}, \ldots, a_{p}-\frac{p+q-1}{2},-b_{1}-n+\frac{p+q-1}{2}, \ldots,-b_{q}-n-\frac{-p+q-1}{2}\right]$

via the Weyl group $W_{K}$. It follows immediately that

$$
\lambda+\rho_{\mathfrak{g}}-2 \rho_{\mathfrak{k}}=\left[a_{1}, \ldots, a_{p},-b_{1}-n, \ldots,-b_{q}-n\right]=\bar{\sigma},
$$

and $\bar{\rho}(\tau)=\mathcal{U}\left(\mathfrak{p}^{+}\right) \cdot \bar{\sigma}$. Since $\bar{\rho}(\tau)$ is a discrete series representation of $U(p, q)$ (see 7]), by Theorem 9.20 in [8, $\lambda$ is the Harish-Chandra parameter of $\bar{\rho}(\tau)$. Moreover, by Theorem 11.178 in $[9], \bar{\rho}(\tau) \simeq \mathrm{A}_{\mathfrak{b}}\left(\lambda-\rho_{\mathfrak{g}}\right)$, where

$$
\lambda-\rho_{\mathfrak{g}}=\left[a_{1}-q, \ldots, a_{p}-q,-b_{1}-n+p, \ldots,-b_{q}-n+p\right] .
$$

By Theorem 2.9 in [21], $\bar{\rho}(\tau)$ occurs in the discrete part of $\mathrm{L}^{2}\left(U(p, q) / S O_{e}(p, q)\right)$, which means $\operatorname{Hom}_{S O_{e}(p, q)}(\bar{\rho}(\tau), 1) \neq 0$. Then there exists a unitary character $\xi^{\prime}$ such that

$$
\operatorname{dim} \operatorname{Hom}_{O(p, q)}\left(\bar{\rho}(\tau), \xi^{\prime}\right)=\operatorname{dim} \operatorname{Hom}_{O(p) \times O(q)}\left(\bar{\sigma}, \xi^{\prime}\right)=1 .
$$

Moreover,

$$
\xi^{\prime} \simeq \begin{cases}\bar{\xi}_{+,-}, & \text {if } n \text { is odd } \\ \bar{\xi}_{+,+}, & \text {if } n \text { is even }\end{cases}
$$

Then $\bar{\xi} \otimes \hat{\xi} \simeq \xi^{\prime}$. It means $\operatorname{dim} \operatorname{Hom}_{O(p, q)}(\bar{\rho}(\tau), \bar{\xi} \otimes \hat{\xi})=1$. By Theorem 4.5

$$
\theta(\xi) \simeq \sum_{\substack{a_{i} \text { are even, } b_{i} \text { are even, } \\ a_{1} \geq \cdots \geq a_{p} \geq 0 \geq-b_{1} \geq \cdots \geq-b_{q}}} \tau_{n}^{\frac{p-q}{2} \mathbf{1}_{n}+\left[a_{1}, \ldots, a_{p}, \mathbf{0}_{n-p-q},-b_{1}, \ldots,-b_{q}\right]} .
$$

Remark 4.7. The representations $\theta(1)$ and $\theta$ (det) have been well studied in [24] and 12 .

Combining Theorems 4.4 and 4.6, we have the following theorem.

Theorem 4.8. Let $\mathcal{O}$ be an admissible Vogan orbit of $\operatorname{Sp}(2 n, \mathbb{R})($ or $M p(2 n, \mathbb{R}))$ corresponding to the signed Young diagram $(+2)^{p}(-2)^{q}(+1)^{2(n-p-q)}$. Then $n>$ $p+q$. Let $\left(x, \chi \otimes \tau_{n}^{\frac{q-p}{2} \mathbf{1}_{n}}\right)$ be the admissible datum given in Proposition 4.2 (or Proposition 4.3). Then there exists a unitary representation $\theta(\xi)$, which is the theta lifting of a unitary character $\xi$ of $\widetilde{O(p, q)}$, such that

$$
\left.\operatorname{Ind}_{K_{\mathbb{C}}(x)}^{K_{\mathbb{C}}}\left(\chi \otimes \tau_{n}^{\frac{q-p}{2}} \mathbf{1}_{n}\right) \simeq(\theta(\xi))^{*}\right|_{K},
$$

and the associated variety $\mathcal{A} \mathcal{V}\left((\theta(\xi))^{*}\right)=\overline{\mathcal{O}}$. If $(p, q) \neq(1,1)$, this representation is unique up to isomorphism.

Proof. Let $\left(x, \chi \otimes \tau_{n}^{\frac{q-p}{2}} \mathbf{1}_{n}\right)$ be an admissible datum of the nilpotent $K_{\mathbb{C}^{-o r b i t}} \mathcal{O}$. Comparing the $K$-module structure of $\operatorname{Ind}_{K_{\mathbb{C}}(x)}^{K_{\mathbb{C}}}\left(\chi \otimes \tau_{n}^{\frac{q-p}{2}} \mathbf{1}_{n}\right)$ with $\theta(\xi)^{*}$, there exists a unitary representation $\theta(\xi)$, which is obtained by the theta lifting of a unitary character $\xi$ of $\widetilde{O(p, q)}$, such that

$$
\left.\theta(\xi)^{*}\right|_{K} \simeq \operatorname{Ind}_{K_{\mathbb{C}}(x)}^{K_{\mathbb{C}}}\left(\chi \otimes \tau_{n}^{\frac{q-p}{2}} \mathbf{1}_{n}\right) .
$$


The associated variety $\mathcal{A} \mathcal{V}\left(\theta(\xi)^{*}\right)$ has been obtained by Przebinda ([18, corollary 7.10 and [19, Theorem 1.4; see also [16, lemma 4.13). Then we have $\mathcal{A} \mathcal{V}\left(\theta(\xi)^{*}\right)=$ $\overline{\mathcal{O}}$. If $(p, q) \neq(1,1)$ and $p q \neq 0$, by Theorem 5.5 in $[6$, this unitary representation $\theta(\xi)$ is unique up to isomorphism. If $p q=0$, the uniqueness property can be obtained by checking the $K$-types structure and the classification of low rank representations.

\section{ACKNOWLEDGEMENTS}

The author would like to express his deepest gratitude to Professor Jing-Song Huang, who has given careful guidance and has kindly supported the author's Ph.D. research. Moreover, the author would also like to thank Professors David Vogan, Jian-Shu Li and Chen-Bo Zhu for their helpful discussions.

\section{REFERENCES}

[1] J. Adams, J.-S. Huang and D.A. Vogan, Functions on the model orbit in $E_{8}$, Represent. Theory 2 (1998), 224-263. MR1628031 (99g:20077)

[2] B. Binegar, On a class of multiplicity-free nilpotent $K_{\mathbb{C}}$-orbits, J. Math. Kyoto Univ. 47 (2007), no. 4, 735-766. MR2413063(2010b:22017)

[3] D. Collingwood and W. McGovern, Nilpotent orbits in semisimple Lie algebras, Van Nostrand Reinhold Mathematics Series. Van Nostrand Reinhold Co., New York, 1993. MR 1251060 (94j:17001)

[4] R. Howe, Transcending classical invariant theory. J. Amer. Math. Soc. 2 (1989), no. 3, 535-552. MR985172 (90k:22016)

[5] R. Howe and C.-B. Zhu, Eigendistributions for orthogonal groups and representations of symplectic groups. J. Reine Angew. Math. 545 (2002), 121-166. MR.1896100 (2003g:20077)

[6] J.-S. Huang and J.-S. Li, Unipotent representations attached to spherical nilpotent orbits. Amer. J. Math. 121 (1999), no. 3, 497-517. MR.1738410 (2000m:22018)

[7] M. Kashiwara and M. Vergne, On the Segal-Shale-Weil representations and harmonic polynomials. Invent. Math. 44 (1978), no. 1, 1-47. MR0463359 (57:3311)

[8] A.W. Knapp, Representation theory of semisimple groups. An overview based on examples. Princeton Mathematical Series, 36. Princeton University Press, Princeton, NJ, 1986. MR.855239 (87j:22022)

[9] A.W. Knapp and D.A. Vogan, Cohomological induction and unitary representations. Princeton Mathematical Series, 45. Princeton University Press, Princeton, NJ, 1995. MR 1330919 (96c:22023)

[10] D.R. King, Classification of spherical nilpotent orbits in complex symmetric space, J. Lie Theory 14 (2004), no. 2, 339-370. MR2066860 (2005e:22012)

[11] B. Kostant, Lie algebra cohomology and the generalized Borel-Weil theorem, Ann. of Math. (2) 74 (1961), 329-387. MR0142696 (26:265)

[12] S. Kudla and S. Rallis, Degenerate principal series and invariant distribution, Israel J. Math. 69 (1990), 25-45. MR1046171

[13] S.L. Lee and C.-B. Zhu, Degenerate principal series and local theta correspondence. Trans. Amer. Math. Soc. 350 (1998), no. 12, 5017-5046. MR.1443883 (99c:22021)

[14] J.-S. Li, Singular unitary representations of classical groups. Invent. Math. 97 (1989), no. 2, 237-255. MR.1001840 (90h:22021)

[15] Kyo Nishiyama, H. Ochiai, K. Taniguchi and H. Yamashita, Nilpotent orbits, associated cycles and Whittaker models for highest weight representations. Astérisque No. 273 (2001). Société Mathématique de France, Paris, 2001. pp. i-vi and 1-163. MR1845713(2002b:22025)

[16] Kyo Nishiyama and C.-B. Zhu, Theta lifting of holomorphic discrete series: the case of $U(n, n) \times U(p, q)$. Trans. Amer. Math. Soc. 353 (2001), no. 8, 3327-3345. MR.1828608 (2002e:22017)

[17] Kyo Nishiyama and C.-B. Zhu, Theta lifting of unitary lowest weight modules and their associated cycles. Duke Math. J. 125 (2004), no. 3, 415-465. MR2166751 (2006f:22007)

[18] T. Przebinda, Characters, dual pairs, and unitary representations. Duke Math. J. 69 (1993), no. 3, 547-592. MR 1208811(94i:22036) 
[19] W. Schmid and K. Vilonen, Characteristic cycles and wave front cycles of representations of reductive Lie groups. Ann. of Math. (2) 151 (2000), no. 3, 1071-1118. MR.1779564 $(2001 \mathrm{j}: 22017)$

[20] D.A. Vogan, Associated varieties and unipotent representations, Harmonic analysis on reductive groups (W. Barker and P. Sally, eds.), 315-388, Birkhäuser, Boston-Basel-Berlin, 1991. MR 1168491 (93k:22012)

[21] D.A. Vogan, Irreducibility of discrete series representations for semisimple symmetric spaces. Representations of Lie groups, Kyoto, Hiroshima, 1986, 191-221, Adv. Stud. Pure Math., 14, Academic Press, Boston, MA, 1988. MR 1039838 (91b:22023)

[22] D.A. Vogan, Representations of reductive Lie groups. Proceedings of the International Congress of Mathematicians, Vol. 1, 2 (Berkeley, Calif., 1986), 245-266, Amer. Math. Soc., Providence, RI, 1987. MR934226 (89h:22034)

[23] D.A. Vogan, The method of coadjoint orbits for real reductive groups. Representation theory of Lie groups (Park City, UT, 1998), 179-238, IAS/Park City Math. Ser., 8, Amer. Math. Soc., Providence, RI, 2000. MR1737729 (2001k:22027)

[24] C.-B. Zhu, Invariant distributions of classical groups. Duke Math. J. 65 (1992), no. 1, 85119. MR1148986 (92k:22022)

[25] C.-B. Zhu, J.-S. Huang, On certain small representations of indefinite orthogonal groups. Represent. Theory 1 (1997), 190-206. MR 1457244 (98j:22026)

Department of Mathematics, Sichuan University, Chengdu, 610064, People's Republic OF CHINA

E-mail address: malyang@scu.edu.cn 